\title{
Parkinson's disease outcomes after intraoperative CT-guided "asleep" deep brain stimulation in the globus pallidus internus
}

\author{
Zaman Mirzadeh, MD, PhD, ${ }^{1}$ Kristina Chapple, PhD, ${ }^{1}$ Margaret Lambert, RN, BSN, ${ }^{1}$ \\ Virgilio G. Evidente, MD, ${ }^{3}$ Padma Mahant, MD, ${ }^{4}$ Maria C. Ospina, MD, ${ }^{4}$ Johan Samanta, MD, ${ }^{4}$ \\ Guillermo Moguel-Cobos, MD, ${ }^{2}$ Naomi Salins, MD, ${ }^{2}$ Abraham Lieberman, MD, ${ }^{2}$ \\ Alexander I. Tröster, PhD, ${ }^{5}$ Rohit Dhall, MD, ${ }^{2}$ and Francisco A. Ponce, MD ${ }^{1}$ \\ Divisions of ${ }^{1}$ Neurological Surgery, ${ }^{2}$ Neurology, and ${ }^{5}$ Clinical Neuropsychology, Barrow Neurological Institute, St. Joseph's \\ Hospital and Medical Center, Phoenix; ${ }^{3}$ Movement Disorders Center of Arizona, Scottsdale; and ${ }^{4}$ Banner Good Samaritan \\ Medical Center, Phoenix, Arizona
}

\begin{abstract}
OBJECTIVE Recent studies show that deep brain stimulation can be performed safely and accurately without microelectrode recording or test stimulation but with the patient under general anesthesia. The procedure couples techniques for direct anatomical targeting on MRI with intraoperative imaging to verify stereotactic accuracy. However, few authors have examined the clinical outcomes of Parkinson's disease (PD) patients after this procedure. The purpose of this study was to evaluate PD outcomes following "asleep" deep brain stimulation in the globus pallidus internus (GPi).
\end{abstract}

METHODS The authors prospectively examined all consecutive patients with advanced PD who underwent bilateral GPi electrode placement while under general anesthesia. Intraoperative CT was used to assess lead placement accuracy. The primary outcome measure was the change in the off-medication Unified Parkinson's Disease Rating Scale motor score 6 months after surgery. Secondary outcomes included effects on the 39-Item Parkinson's Disease Questionnaire (PDQ-39) scores, on-medication motor scores, and levodopa equivalent daily dose. Lead locations, active contact sites, stimulation parameters, and adverse events were documented.

RESULTS Thirty-five patients ( 24 males, 11 females) had a mean age of 61 years at lead implantation. The mean radial error off plan was $0.8 \mathrm{~mm}$. Mean coordinates for the active contact were $21.4 \mathrm{~mm}$ lateral, $4.7 \mathrm{~mm}$ anterior, and $0.4 \mathrm{~mm}$ superior to the midcommissural point. The mean off-medication motor score improved from 48.4 at baseline to 28.9 ( $40.3 \%$ improvement) at 6 months $(p<0.001)$. The PDQ-39 scores improved $(50.3$ vs $42.0 ; p=0.03)$, and the levodopa equivalent daily dose was reduced ( $1207 \mathrm{vs} 1035 \mathrm{mg} ; \mathrm{p}=0.004$ ). There were no significant adverse events.

CONCLUSIONS Globus pallidus internus leads placed with the patient under general anesthesia by using direct anatomical targeting resulted in significantly improved outcomes as measured by the improvement in the off-medication motor score at 6 months after surgery.

Clinical trial registration no.: NCT01997398 (clinicaltrials.gov)

http://thejns.org/doi/abs/10.3171/2015.4.JNS1550

KEY WORDS deep brain stimulation; Parkinson's disease; stereotactic accuracy; intraoperative imaging; functional neurosurgery

$\mathrm{O}$ VER the past 30 years, deep brain stimulation (DBS) has evolved into a mainstream therapy for patients with Parkinson's disease (PD) that is supported by Level 1 evidence. ${ }^{5,6,25,31,32}$ The clinical efficacy of DBS depends on appropriate lead placement within the targeted structure. , $, 20,22$ Stereotactic targeting was initially per- formed via "indirect" methods using Cartesian coordinates of the targeted structure (ventral intermediate nucleus, subthalamic nucleus $[\mathrm{STN}],{ }^{25}$ or globus pallidus internus [GPi]) relative to the midcommissural point on stereotactic anatomical atlases..$^{24}$ Electrophysiological mapping and test stimulation would then be performed on the patient

ABBREVIATIONS ADL = activity of daily living; DBS = deep brain stimulation; FGATIR = fast gray matter acquisition T1 inversion recovery; GPi = globus pallidus internus; MDS = International Parkinson and Movement Disorder Society; MER = microelectrode recording; PD = Parkinson's disease; PDQ-39 = 39-Item Parkinson's Disease Questionnaire; STN = subthalamic nucleus; UPDRS-III = Unified Parkinson's Disease Rating Scale Part III.

SUBMITTED January 8, 2015. ACCEPTED April 9, 2015.

INCLUDE WHEN CITING Published online October 9, 2015; DOI: 10.3171/2015.4.JNS1550. 
while he or she was awake to refine final lead placement. Studies of these methods with postoperative imaging to assess final lead location in the $\mathrm{STN}^{3,11,15,20,23,26,33}$ or $\mathrm{GPi}^{5,14,28,30}$ revealed important correlations between the precise anatomical lead position and clinical outcome. These data coupled with improved MRI resolution led the way for "direct" targeting, wherein the targeted structure is directly visualized on MRI. Direct targeting now largely supplants the older indirect method; however, the adjunctive use of intraoperative electrophysiology and test stimulation as tools to determine and verify appropriate lead position remains the standard of care at most centers.

Now, as the availability of intraoperative imaging has increased, the most recent iteration of the DBS procedure performed by some groups relies entirely on stereotactic anatomical accuracy, without the use of microelectrode recording (MER) or test stimulation. Recent studies show that DBS can be performed safely and accurately with intraoperative confirmation of lead position based on intraoperative $\mathrm{CT}^{4,17}$ or interventional MRI. ${ }^{27}$ This so-called asleep DBS procedure can be performed with the patient under general anesthesia, which may be better tolerated by patients, may render the therapy more attractive to good candidates for DBS who may otherwise not consider surgery, and avoids the significant added risk of MER. ${ }^{1,10,35}$ Despite growing interest in the asleep DBS technique, however, few clinical outcome studies in PD patients have been performed for STN targeting ${ }^{9,21}$ and none for GPi targeting.

To address this deficiency in the literature, we present the first clinical outcome data from a prospective consecutive cohort of PD patients undergoing bilateral GPi asleep DBS.

\section{Methods}

\section{Patients and Clinical Outcome Scales}

All consecutive PD patients undergoing bilateral GPi DBS under general anesthesia in the period from May 2012 to November 2013 were prospectively enrolled in this study. Potential candidates were evaluated by a multidisciplinary team, including a fellowship-trained movement disorders neurologist, neuropsychologist, and neurosurgeon. Patients were considered appropriate for DBS if they had a diagnosis of idiopathic PD with 1) an adequate response to dopaminergic medication and 2) disabling motor fluctuations, dyskinesia, or severe functional impairment despite medical management. The decision to perform the surgery under general anesthesia was based on patient preference, the referring neurologist's approval, and an informed consent process that made clear to patients and their families that "awake" surgery is the more accepted and established method and that explicitly reviewed the advantages of awake surgery and the paucity of data supporting asleep surgery.

A movement disorders clinician who had completed the International Parkinson and Movement Disorder Society's (MDS's) Unified Parkinson's Disease Rating Scale (UPDRS) training performed prospective baseline and 6-month postoperative assessments of motor function using the MDS-modified UPDRS Part III (UPDRS-III; mo- tor examination) on patients during both off-medication (PD medications withheld for 12 hours) and on-medication states. Patients self-assessed their quality of life using the 39-Item Parkinson's Disease Questionnaire (PDQ-39) at baseline and 6 months after surgery. The primary outcome measure was change in off-medication UPDRS-III score from baseline to 6 months postoperatively. Secondary outcomes included changes at 6 months in PDQ-39 scores, on-medication UPDRS-III scores, and levodopa equivalent daily dose; DBS lead location and active contact sites; DBS stimulation parameters; and adverse events.

The study protocol was approved by the Institutional Review Board at St. Joseph's Hospital and Medical Center in Phoenix, Arizona. The patients gave written approval on informed consent documentation to have their health information recorded and used for research. Patients were de-identified for the analysis. This study was registered with the ClinicalTrials.gov database (http://clinicaltrials. gov), and its registration no. is NCT01997398.

\section{Surgical Procedure}

Direct targeting was performed using data from preoperative MR images (fast gray matter acquisition T1 inversion recovery [FGATIR], 1-mm slice; ${ }^{29}$ or proton density sequence, 2-mm slice), which were coregistered with intraoperative CT images for stereotactic registration. Images were obtained on a Siemens or General Electric 3-T MRI unit. The targeting method consisted of selecting a point within the GPi at the midcommissural plane, typically starting $4.5 \mathrm{~mm}$ anterior to the midcommissural point, and refining the target based on individual anatomy while staying $3.6 \mathrm{~mm}$ away from the internal capsule. (This method is based on work by Starr et al., specifically the data shown in Fig. 6 and Table 3 of that study. ${ }^{28}$ ) Entry point and trajectory were then determined, with a parasagittal approach attempted at or anterior to the coronal suture, adjusted to enter a gyrus and avoid sulci. The trajectory was then extended ventrally to the level of the optic tract.

A CereTom portable CT scanner (NeuroLogica) was used for intraoperative CT image acquisition, and Medtronic Framelink software was used for image coregistration. Leads were placed using frameless (Nexframe; 22 leads/11 patients) or frame-based (Leksell frame; 48 leads/24 patients) stereotaxy with the patient under general anesthesia. The initial use of frameless stereotaxy was based on our adoption of the technique described by Burchiel et al. ${ }^{4}$ Our subsequent realization that stereotactic registration of the Leksell frame was possible with the CereTom scanner led us to return to frame-based stereotaxy. (A later review of our stereotactic data for asleep DBS revealed a small but significant improvement in accuracy with the frame-based, as compared with the frameless, stereotaxy, ${ }^{17}$ as well as a reduction in total operative time by 1.1 hours. ${ }^{16}$ ) After lead placement, CT images were obtained intraoperatively to determine accuracy by 2 measurements: the Euclidean error between the principal contact and the planned target, and the radial error between the principal contact and the planned trajectory. Leads placed with a radial error greater than $2 \mathrm{~mm}$ were repositioned. Deep brain stimulation internal pulse generators were placed 7-14 days after lead implantation. 


\section{Deep Brain Stimulation Programming}

Neurostimulator programming was initiated by the patients' neurologists 1-2 weeks after pulse generator implantation. Electrode contacts were separately tested in the unipolar mode, and voltage-limiting adverse effects and improvements in PD symptoms were noted and used to set chronic stimulation parameters. There were no formal guidelines for programming, and final settings were largely selected based on the treating neurologists' clinical assessments and preference. Follow-up programming visits were scheduled based on the preference of the treating neurologist.

\section{Statistical Analysis}

Frequencies and descriptive statistics were computed on all variables. Differences between baseline and 6-month postoperative scores were assessed using a series of repeated-measures general linear models with a single within-subjects factor and no between-subjects factors. SPSS version 21 (IBM Corp.) was used for all analyses. Probability values presented are 2-tailed and considered significant when less than 0.05 .

\section{Results}

\section{Patient Characteristics}

Thirty-five consecutive PD patients (24 men, 11 women) underwent bilateral GPi asleep DBS in the study period. The average age at lead implantation was 61.1 years, and the average duration of PD at that point was 10.7 years. The most disabling PD symptoms for this cohort included motor fluctuations (31 patients [89\%]), dyskinesias (25 patients [71\%]), tremor (22 patients [63\%]), insomnia (12 patients [34\%]), behavioral changes (10 patients [29\%]), and medication side effects (3 patients [9\%]).

\section{Clinical Outcomes}

Outcomes analysis was performed on 34 patients with complete data. One patient took PD medications 3 hours prior to the 6-month on-off motor evaluation, precluding off-medication testing, and was therefore excluded from analysis. The primary outcome measure, off-medication UPDRS-III score, improved from a mean $( \pm$ standard deviation) $48.4 \pm 13.8$ at baseline to $28.9 \pm 12.5(40.3 \%$ improvement) at 6 months after surgery $(p<0.001)$, representing a large effect size (partial eta-squared $=0.52$ ). The mean on-medication UPDRS-III score showed no significant change at 6 months $(p=0.73)$. The mean UPDRSIII scores were compared in 3 treatment states tested at 6 months postoperatively: off-medication/off-stimulation $(46.3 \pm 16.1)$ versus off-medication/on-stimulation (28.9 $\pm 12.5)$ versus on-medication/on-stimulation $(21.2 \pm 12.4)$ scores. The overall repeated-measures model showed statistically significant differences among these 3 states with a quadratic effect emerging ( $p<0.001$; Fig. 1 upper). Pairwise comparisons made with the Bonferroni adjustment among these 3 states were all significant $(\mathrm{p}<0.001)$. There were no significant differences in motor scores between baseline off-medication (48.4) and 6-month postoperative off-medication/off-stimulation (46.3). There were no sta-
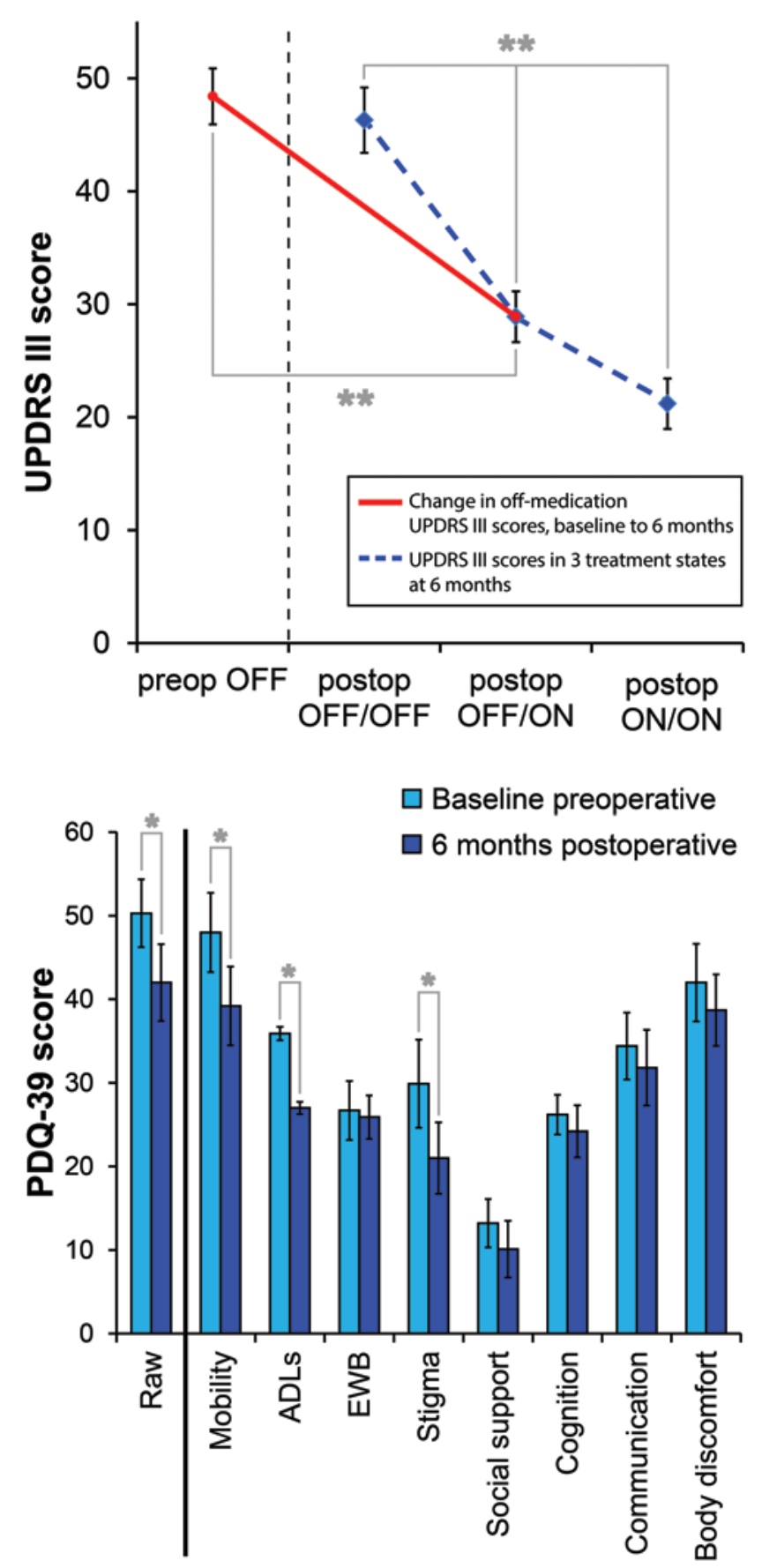

FIG. 1. Mean ( \pm standard error) clinical outcome scores at baseline and 6 months after asleep DBS. ${ }^{*} p<0.05 ;{ }^{* *} p<0.001$. Upper: Change in off-medication UPDRS-III score (solid red line) and scores in 3 treatment states postoperatively (dotted blue line, postop OFF/OFF: off medication/off stimulation; postop OFF/ON: off medication/on stimulation; postop ON/ON: on medication/on stimulation). Lower: The PDQ-39 raw and domain scores. EWB = emotional well-being. Figure is available in color online only.

tistically significant differences in motor scores between patients who had undergone frame-based versus frameless stereotaxy $(\mathrm{p}=0.50)$.

Raw PDQ-39 scores improved significantly from 50.3 \pm 20.7 at baseline to $42.0 \pm 23.5$ at 6 months postoperative- 
ly $($ mean $\pm \mathrm{SD}, \mathrm{p}=0.03$; partial eta-squared $=0.17$ ). Assessing specific domains within the PDQ-39 at 6 months, we found significant improvements in mobility $(48.0 \pm$ 24.2 vs $39.2 \pm 24, \mathrm{p}=0.02$; partial eta-squared $=0.20$ ), activities of daily living (ADLs; $35.9 \pm 4.1$ vs $27.0 \pm 3.7$, $\mathrm{p}=0.01$; partial eta-squared $=0.26)$, and stigma $(29.9 \pm$ 26.9 vs $21.0 \pm 21.8, \mathrm{p}=0.01$; partial eta-squared $=0.22$ ), with no change in the other domains (Fig. 1 lower). Levodopa equivalent daily dose decreased by an average of $14 \%$, from $1207 \pm 482 \mathrm{mg}$ at baseline to $1035 \pm 478 \mathrm{mg}$ at 6 months (mean $\pm \mathrm{SD}, \mathrm{p}=0.004$ ), corresponding to a small effect size (partial eta-squared $=0.22$ ). By 6 months after surgery, 22 left-sided leads (63\%) and 21 right-sided leads $(60 \%)$ had been programmed with monopolar settings, with the remaining leads (13 left, 14 right) having bipolar settings. Average stimulation parameters were $3.3 \mathrm{~V}$, $118 \mu \mathrm{sec}, 128 \mathrm{~Hz}$ for left-sided leads and $3.2 \mathrm{~V}, 129 \mu \mathrm{sec}$, $115 \mathrm{~Hz}$ for right-sided leads. There were no perioperative adverse events.

\section{Lead Location and Stereotactic Accuracy}

For all leads combined, the average Euclidean error was $1.2 \mathrm{~mm}$ and the average radial error was $0.8 \mathrm{~mm}$ off plan. All 35 left-sided leads and 31 right-sided leads were placed with single brain penetrations, with the remaining 4 right-sided leads placed with 2 passes each. Lead repositioning was based purely on stereotactic error determined on postimplantation intraoperative CT: leads with a radial error greater than $2 \mathrm{~mm}$ were repositioned via a $2-\mathrm{mm}$ offset (StarDrive, FHC Inc.) directed opposite the direction of the error. Average lead coordinates, measured at the position of contact 0 , were $-20.50,2.50,-3.85$ for leftsided leads, with a Euclidean error of $1.2 \mathrm{~mm}$ and a radial error of $0.9 \mathrm{~mm}$ compared with plan, and 21.16, 2.64, -3.75 for right-sided leads, with a Euclidean error of $1.1 \mathrm{~mm}$ and a radial error of $0.7 \mathrm{~mm}$. The average coronal trajectory angle was $8.0^{\circ}$, and the average sagittal trajectory angle was $62.2^{\circ}$ for all leads. Average coordinates for the active contact were -21.1, 4.7, 0.7 for left-sided leads and 21.6, 4.6, 0.1 for right-sided leads (Fig. 2). Average procedure time was $2.05 \pm 0.60$ hours (range $1.22-3.50$ hours), and average total operating room time was $3.54 \pm 0.69$ hours (range 2.53-5.10 hours).

\section{Discussion}

In this prospective study of 35 consecutive PD patients undergoing bilateral GPi asleep DBS, there was a significant improvement of $40.3 \%$ in the off-medication UPDRS motor score at 6 months after surgery. The PDQ-39 raw scores were also significantly improved (16.5\%), with the domains of mobility, ADLs, and stigma responsible for this improvement. There was a small but significant decrease (14\%) in levodopa equivalent daily dose, similar to what has been reported for GPi stimulation (3\% in Anderson et al., ${ }^{2} 18 \%$ in Follett et al. ${ }^{8}$ ). Lead placement using intraoperative CT for confirmation of stereotactic accuracy, without MER or test stimulation, was performed with submillimeter radial error $(0.8 \mathrm{~mm})$.

Motor outcomes in our study are comparable to those published in prior studies using traditional MER-guided

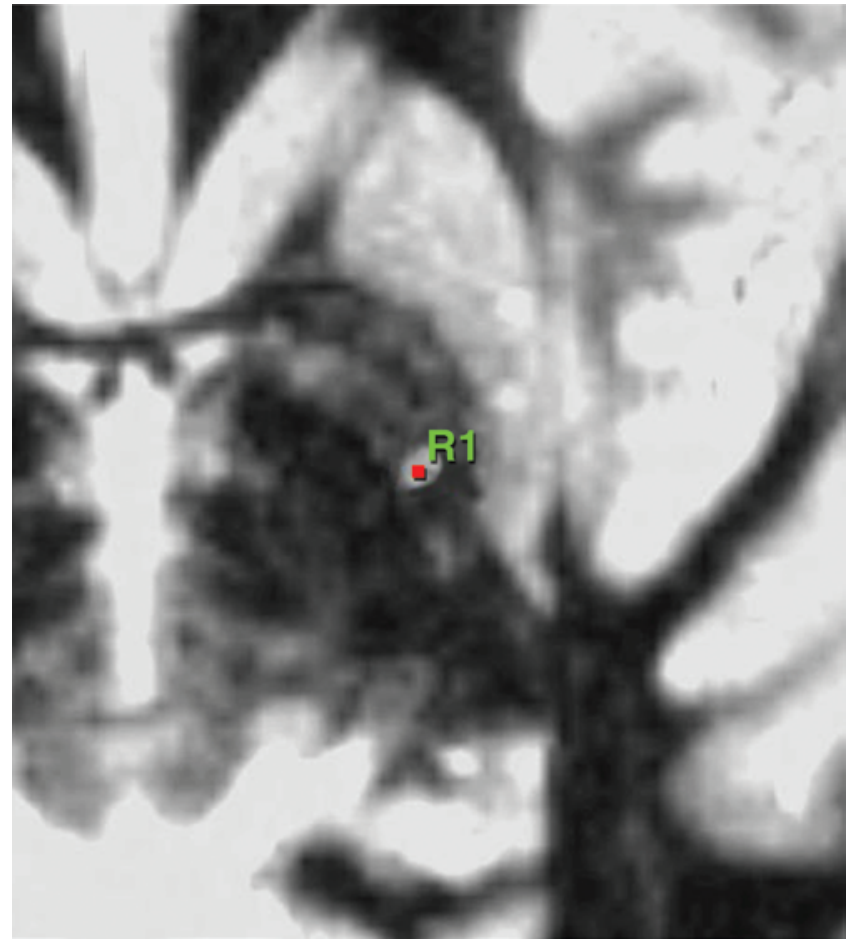

FIG. 2. Computed tomography-MR fusion image comprising a preoperative FGATIR MRI sequence merged with the intraoperative postimplantation CT to show right active contact site. R1 = contact 1 on right. Figure is available in color online only.

techniques for lead placement in the GPi. In one large multicenter trial of DBS for PD, the authors found that bilateral GPi stimulation resulted in an average improvement of 33\% in off-medication UPDRS-III scores at 6 months. ${ }^{5}$ The Veterans Affairs study ${ }^{8}$ demonstrated a $28.2 \%$ improvement and the COMPARE trial ${ }^{19}$ reported a $26.6 \%$ improvement in off-medication UPDRS motor scores with pallidal stimulation at similar follow-up times. Differences in the precise degree of motor improvement in these studies may be due to multiple factors, including severity of PD prior to surgery, unilateral versus bilateral stimulation, and the timing of motor evaluation postoperatively; however, overall motor improvement among patients in these studies and in the current study is largely comparable. Similarly, a prospective study of bilateral STN asleep DBS using an interventional MRI technique revealed motor improvement (49.4\%) comparable to that in a previous study using traditional MER-guided STN lead implantation. ${ }^{21}$

Interventional MRI-guided DBS offers an alternative asleep technique for direct anatomically based placement of DBS electrodes without electrophysiological mapping. ${ }^{12,13,21,27}$ In comparison with our intraoperative CT-guided technique, performing electrode implantation in the interventional MRI suite allows for near real-time visualization of not only the lead or targeting cannula, but also the intended target. ${ }^{12}$ This avoids the requirement for a registration scan and the associated error due to CT-MR image fusion. However, the MRI-based technique incurs additional costs, including those associated with disposable instruments and MRI utilization, and has longer re- 
ported surgical times ( 3 hours for bilateral lead placement in the experience of Larson et al., ${ }^{13}$ as compared with 2 hours in our current study). In addition, we recently reported that the error associated with CT-MR image fusion in our technique is at least partially, if not completely, offset by the error in determining the exact lead or targeting cannula location by MRI, which is associated with a larger metal artifact than CT. ${ }^{17}$ In the end, each technique has its merits and limitations, but both have now shown safe, accurate, and efficacious lead placement.

There is mounting evidence that DBS can be performed safely and effectively under general anesthesia, $4,17,21,27$ with motor outcomes comparable to those obtained after traditional MER-guided methods. So-called asleep and awake DBS are complementary approaches, each with inherent assumptions about optimal lead placement. The use of MER to guide lead placement relies on an "admitted assumption that the physiologically defined optimal target is sufficiently predictive of subsequent clinical response."18 Likewise, the use of intraoperative imaging to guide lead placement in asleep DBS relies on an admitted assumption that the anatomically defined optimal target is sufficiently predictive of the subsequent clinical response. While there is a longer history of MER-guided electrode placement and only more recent experience with imageguided techniques, there is no Level 1 evidence supporting the use of one method over the other. ${ }^{34,35}$ If optimal lead placement can be performed anatomically with stereotactic accuracy as the surgical end point, then performing surgery on awake patients off medication for MER and test stimulation may be reserved for cases with unique anatomical constraints or to verify lead placement in the absence of intraoperative imaging. General anesthesia, in and of itself, is not a necessary condition for the asleep approach insofar as the techniques used to place leads and verify accuracy do not require the patient to be under general anesthesia. In fact, for awake cases, we are increasingly using the full-gantry intraoperative CT (BodyTom, Neurologica) to verify lead position immediately after test stimulation, with the Leksell arc still in place and with the stylet still within the DBS lead. This is particularly useful in the setting of ambiguous recordings or equivocal clinical responses (for example, non-tremor-dominant PD), providing another piece of intraoperative data upon which to base final lead placement.

There are important limitations in this study that must be considered. Patients and evaluating clinicians were not blinded to the patient's treatment state during testing, so a placebo effect cannot be excluded. Patients were not randomized to undergo asleep DBS versus traditional MERguided DBS; treatment was instead selected based on patient preference. The number of patients presented here is small and all procedures were performed by a single surgeon, which could affect the generalizability of the results. The UPDRS motor scores were not separately tallied for left and right, which precluded us from analyzing the relationship between motor improvement and either active contact site or lead placement error. Finally, the 35 patients in this study were referred for DBS and later had DBS programming performed by 12 different neurologists, each probably with a unique approach to programming. Motor improvements after DBS depend on optimization of DBS programming coupled with medical management. The lack of standardization when choosing active contacts and stimulation parameters partially confounds the analysis of the ideal active contact site for optimal motor response.

\section{Conclusions}

In summary, this study demonstrates that anatomical targeting and intraoperative CT-guided DBS lead implantation in the GPi under general anesthesia can achieve motor outcomes comparable to traditional MER-guided methods in PD patients.

\section{References}

1. Abosch A, Timmermann L, Bartley S, Rietkerk HG, Whiting D, Connolly PJ, et al: An international survey of deep brain stimulation procedural steps. Stereotact Funct Neurosurg 91:1-11, 2013

2. Anderson VC, Burchiel KJ, Hogarth P, Favre J, Hammerstad JP: Pallidal vs subthalamic nucleus deep brain stimulation in Parkinson disease. Arch Neurol 62:554-560, 2005

3. Anheim M, Batir A, Fraix V, Silem M, Chabardès S, Seigneuret E, et al: Improvement in Parkinson disease by subthalamic nucleus stimulation based on electrode placement: effects of reimplantation. Arch Neurol 65:612-616, 2008

4. Burchiel KJ, McCartney S, Lee A, Raslan AM: Accuracy of deep brain stimulation electrode placement using intraoperative computed tomography without microelectrode recording. J Neurosurg 119:301-306, 2013

5. Deep-Brain Stimulation for Parkinson's Disease Study Group: Deep-brain stimulation of the subthalamic nucleus or the pars interna of the globus pallidus in Parkinson's disease. N Engl J Med 345:956-963, 2001

6. Deuschl G, Schade-Brittinger C, Krack P, Volkmann J, Schäfer H, Bötzel K, et al: A randomized trial of deep-brain stimulation for Parkinson's disease. N Engl J Med 355:896908, 2006

7. Ellis TM, Foote KD, Fernandez HH, Sudhyadhom A, Rodriguez RL, Zeilman P, et al: Reoperation for suboptimal outcomes after deep brain stimulation surgery. Neurosurgery 63:754-761, 2008

8. Follett KA, Weaver FM, Stern M, Hur K, Harris CL, Luo P, et al: Pallidal versus subthalamic deep-brain stimulation for Parkinson's disease. N Engl J Med 362:2077-2091, 2010

9. Foltynie T, Zrinzo L, Martinez-Torres I, Tripoliti E, Petersen E, Holl E, et al: MRI-guided STN DBS in Parkinson's disease without microelectrode recording: efficacy and safety. J Neurol Neurosurg Psychiatry 82:358-363, 2011

10. Hariz MI: Safety and risk of microelectrode recording in surgery for movement disorders. Stereotact Funct Neurosurg 78:146-157, 2002

11. Lanotte MM, Rizzone M, Bergamasco B, Faccani G, Melcarne A, Lopiano L: Deep brain stimulation of the subthalamic nucleus: anatomical, neurophysiological, and outcome correlations with the effects of stimulation. J Neurol Neurosurg Psychiatry 72:53-58, 2002

12. Larson PS, Starr PA, Bates G, Tansey L, Richardson RM, Martin AJ: An optimized system for interventional magnetic resonance imaging-guided stereotactic surgery: preliminary evaluation of targeting accuracy. Neurosurgery 70 (1 Suppl Operative): $95-103,2012$

13. Larson PS, Starr PA, Ostrem JL, Galifianakis NB, Palenzuela MSL, Martin AJ: Application accuracy of a second generation interventional MRI stereotactic platform: initial experience in 101 DBS electrode implantations. Neurosurgery 60 (Suppl 1):187, 2013 (Abstract \#203) 
14. Lehman RM, Augustine JR: Evolution and rebirth of functional stereotaxy in the subthalamus. World Neurosurg 80:521-533, 2013

15. McClelland S III, Ford B, Senatus PB, Winfield LM, Du YE, Pullman SL, et al: Subthalamic stimulation for Parkinson disease: determination of electrode location necessary for clinical efficacy. Neurosurg Focus 19(5):E12, 2005

16. Mirzadeh Z, Chapple K, Lambert M, Dhall R, Ponce FA: Stereotactic technique determines accuracy and efficiency in "asleep" DBS. Stereotact Funct Neurosurg 92 (Suppl 1):36, 2014 (Abstract \#148)

17. Mirzadeh Z, Chapple K, Lambert M, Dhall R, Ponce FA: Validation of CT-MRI fusion for intraoperative assessment of stereotactic accuracy in DBS surgery. Mov Disord 29:17881795,2014

18. Montgomery EB Jr: Microelectrode targeting of the subthalamic nucleus for deep brain stimulation surgery. Mov Disord $27: 1387-1391,2012$

19. Okun MS, Fernandez HH, Wu SS, Kirsch-Darrow L, Bowers $\mathrm{D}$, Bova F, et al: Cognition and mood in Parkinson's disease in subthalamic nucleus versus globus pallidus interna deep brain stimulation: the COMPARE trial. Ann Neurol 65:586595,2009

20. Okun MS, Tagliati M, Pourfar M, Fernandez HH, Rodriguez RL, Alterman RL, et al: Management of referred deep brain stimulation failures: a retrospective analysis from 2 movement disorders centers. Arch Neurol 62:1250-1255, 2005

21. Ostrem JL, Galifianakis NB, Markun LC, Grace JK, Martin AJ, Starr PA, et al: Clinical outcomes of PD patients having bilateral STN DBS using high-field interventional MR-imaging for lead placement. Clin Neurol Neurosurg 115:708-712, 2013

22. Richardson RM, Ostrem JL, Starr PA: Surgical repositioning of misplaced subthalamic electrodes in Parkinson's disease: location of effective and ineffective leads. Stereotact Funct Neurosurg 87:297-303, 2009

23. Saint-Cyr JA, Hoque T, Pereira LC, Dostrovsky JO, Hutchison WD, Mikulis DJ, et al: Localization of clinically effective stimulating electrodes in the human subthalamic nucleus on magnetic resonance imaging. J Neurosurg 97:1152-1166, 2002

24. Schaltenbrand G, Wahren W: Atlas for Stereotaxy of the Human Brain. Stuttgart: Thieme, 1977

25. Schuepbach WM, Rau J, Knudsen K, Volkmann J, Krack P, Timmermann L, et al: Neurostimulation for Parkinson's disease with early motor complications. N Engl J Med 368:610-622, 2013

26. Starr PA, Christine CW, Theodosopoulos PV, Lindsey N, Byrd D, Mosley A, et al: Implantation of deep brain stimulators into the subthalamic nucleus: technical approach and magnetic resonance imaging-verified lead locations. J Neurosurg 97:370-387, 2002

27. Starr PA, Martin AJ, Ostrem JL, Talke P, Levesque N, Larson PS: Subthalamic nucleus deep brain stimulator placement using high-field interventional magnetic resonance imaging and a skull-mounted aiming device: technique and application accuracy. J Neurosurg 112:479-490, 2010
28. Starr PA, Turner RS, Rau G, Lindsey N, Heath S, Volz M, et al: Microelectrode-guided implantation of deep brain stimulators into the globus pallidus internus for dystonia: techniques, electrode locations, and outcomes. J Neurosurg 104:488-501, 2006

29. Sudhyadhom A, Haq IU, Foote KD, Okun MS, Bova FJ: A high resolution and high contrast MRI for differentiation of subcortical structures for DBS targeting: the Fast Gray Matter Acquisition T1 Inversion Recovery (FGATIR). Neuroimage 47 (Suppl 2):T44-T52, 2009

30. Volkmann J, Sturm V, Weiss P, Kappler J, Voges J, Koulousakis A, et al: Bilateral high-frequency stimulation of the internal globus pallidus in advanced Parkinson's disease. Ann Neurol 44:953-961, 1998

31. Weaver FM, Follett K, Stern M, Hur K, Harris C, Marks WJ $\mathrm{Jr}$, et al: Bilateral deep brain stimulation vs best medical therapy for patients with advanced Parkinson disease: a randomized controlled trial. JAMA 301:63-73, 2009

32. Williams A, Gill S, Varma T, Jenkinson C, Quinn N, Mitchell R, et al: Deep brain stimulation plus best medical therapy versus best medical therapy alone for advanced Parkinson's disease (PD SURG trial): a randomised, open-label trial. Lancet Neurol 9:581-591, 2010

33. Zonenshayn M, Sterio D, Kelly PJ, Rezai AR, Beric A: Location of the active contact within the subthalamic nucleus (STN) in the treatment of idiopathic Parkinson's disease. Surg Neurol 62:216-226, 2004

34. Zrinzo L, Foltynie T, Limousin P, Hariz M: Image-guided and image-verified deep brain stimulation. Mov Disord 28:254, 2013 (Letter)

35. Zrinzo L, Foltynie T, Limousin P, Hariz MI: Reducing hemorrhagic complications in functional neurosurgery: a large case series and systematic literature review. J Neurosurg 116:84-94, 2012

\section{Disclosure}

Dr. Ponce is a consultant for Medtronic and has received clinical or research support from Barrow Center for Neuromodulation for the study described.

\section{Author Contributions}

Conception and design: Ponce, Mirzadeh. Acquisition of data: Ponce, Mirzadeh, Lambert, Tröster, Dhall. Analysis and interpretation of data: Ponce, Mirzadeh, Chapple. Drafting the article: Ponce, Mirzadeh. Critically revising the article: Ponce, Mirzadeh. Reviewed submitted version of manuscript: Ponce, Mirzadeh. Statistical analysis: Mirzadeh, Chapple. Administrative/technical/ material support: Lambert, Evidente, Mahant, Ospina, Samanta, Moguel-Cobos, Salins, Lieberman. Study supervision: Ponce.

\section{Correspondence}

Francisco A. Ponce, c/o Neuroscience Publications, Barrow Neurological Institute, St. Joseph's Hospital and Medical Center, 350 W. Thomas Rd., Phoenix, AZ 85013.email: neuropub@ dignityhealth.org. 